\title{
Avaliação evolutiva da espirometria na fibrose cística*
}

\author{
ElenaRA DA FonseCA ANDRADE ${ }^{1}$, Deisi Letícia Oliveira da FonseCA ${ }^{2}$,

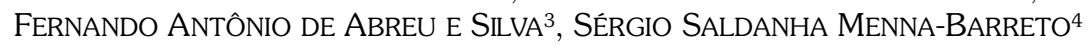

Objetivo do estudo: Avaliar o padrão da evolução dos fluxos e volumes pulmonares dinâmicos dos pacientes com fibrose cística (FC), assim como analisar a relação entre a gravidade do comprometimento ventilatório com a idade, sexo, genótipo e colonização pulmonar. Material e método: Na Unidade de Pneumologia Infantil e Serviço de Pneumologia do Hospital de Clínicas de Porto Alegre, foram avaliadas 243 espirometrias de 52 pacientes portadores de FC maiores de quatro anos realizadas no período de 1987 a 1999. Do prontuário médico foram extraídas informações sobre idade ao diagnóstico, pesquisa genética, colonização brônquica e os valores absolutos e percentuais para fluxos e volumes da melhor espirometria de cada ano. Resultados: A idade dos pacientes variou de quatro a 26 anos (média $=13,04 \pm 4,82$ ). Dos 52 pacientes, 49 (94\%) tiveram pelo menos uma cultura de escarro com $P$. aeruginosa, 45 (86\%) com $S$. aureus e 13 (25\%) com $B$. cepacia. Na avaliação inicial dos quatro aos seis anos $(n=40)$, os valores médios da CVF e do $\mathrm{VEF}_{1}$ foram de $114,24 \%$ e 112,25\%, respectivamente. A média da CVF manteve-se acima do normal até os 18 anos, quando apresentou queda súbita para 67,2\% $(\mathrm{p}=0,0002)$. A média do $\mathrm{VEF}_{1}$ atingiu valores abaixo de $80 \%$ aos dez anos, estando em $50 \%$ aos 18 anos $(\mathrm{p}<0,00001)$. A relação $\mathrm{VEF}_{1} / \mathrm{CVF} \%$, índice mais sensivel de obstrução, mostrou-se diminuída já na primeira avaliação, sendo $85 \%$ entre os quatro e seis anos, com declínio lento progressivo, chegando a $63 \%$ aos 18 anos. Os valores médios do $\mathrm{FEF}_{50}, \mathrm{FEF}_{75}$ e FMEF inicialmente foram normais $(94 \%, 80 \%$ e $90 \%$, respectivamente), mostraram queda maior e mais precoce, estatisticamente significativa, atingindo valores médios aos 18 anos de $37 \%, 12,5 \%$ e $19 \%$, respectivamente. Embora houvesse tendência para fluxos terminais mais baixos para as meninas, as diferenças não tiveram valor estatístico. Também não foram detectadas correlações estatisticamente significativas entre a função pulmonar e o germe colonizador da via aérea ou o genótipo. Conclusão: O padrão evolutivo das alterações funcionais pulmonares encontrado nos pacientes está de acordo com o descrito na literatura, onde predomina o distúrbio ventilatório obstrutivo com redução precoce dos fluxos terminais e acometimento tardio da CVF.

(J Pneumol 2001;27(3):130-136)

\section{Spirometry evolution assessment of cystic fibrosis}

Objectives: To evaluate the evolution pattern of dynamic pulmonary flow and volume in cystic fibrosis patients and analyze the relation between the severity of ventilatory attacks and age, gender, genotype and pulmonary colonization. Methods: At the Pediatric Pulmonary Unit and Pulmonary Service of Hospital de Clinicas of Porto Alegre, 243 spirometries performed between 1987 and 1999 in 52 cystic fibrosis patients older than four years of age were reviewed. From the patients' medical records the following informations were extracted: age at diagnosis, genetic data, bronchial colonization and absolute as well as percent values of the flows and volumes of the best annual spirometry. Results: The age of the patients ranged from four to 26 years

* Trabalho realizado na Universidade Federal do Rio Grande do Sul.

1. Médica Assistente da Unidade de Pneumologia Infantil do Hospital de Clínicas de Porto Alegre; Mestranda do Curso de Pós-Graduação.

2. Doutouranda do Curso de Medicina.

3. Professor Adjunto do Departamento de Pediatria; Chefe da Unidade de Pneumologia Infantil do Hospital de Clínicas de Porto Alegre.
4. Professor Titular do Departamento de Medicina Interna; Chefe do Serviço de Pneumologia e da Unidade de Fisiologia Pulmonar do Hospital de Clínicas de Porto Alegre.

Endereço para correspondência - Dra Elenara da Fonseca Andrade, Unidade de Pneumologia Infantil do Hospital de Clínicas de Porto Alegre, Rua Ramiro Barcelos, 2.350, sala 1.131. Tel. (51) 316-8515; Email: eandrade@terra.com.br

Recebido para publicação em 16/6/00. Reapresentado em 14/ 12/00. Aprovado, após revisão, em 15/2/01. 
(mean $=13.04 \pm 4.82)$. Out of the 52 patients, $49(94 \%)$ had at least one sputum culture with $\mathrm{P}$. aeruginosa, 45 (86\%) with S. aureus and 13 (25\%) with B. cepacia. At initial evaluation, that included patients four to six years old $(n=40)$, mean values for FVC and $F E V_{1}$ were $114.24 \%$ and $112.25 \%$, respectively. Mean FVC remained above baseline until 18 years of age, when it abruptly dropped to $67.2 \%(p=0.0002)$. Mean FEV $V_{1}$ values dropped to less than $80 \%$ at ten years and was $50 \%$ at 18 years $(p<0.00001)$. The $F E V_{1} / F V C \%$ relationship, the most obstruction sensitive index, was observed to be reduced as early as initial evaluation. It was $85 \%$ between four and six years and gradually decreased to $63 \%$ at 18 years. The mean values of $F E F_{50}, F E F_{75}$ and FMEF were initially normal $(94 \%, 80 \%$ and $90 \%$, respectively), showed a greater and earlier statistically significant decline, and reached to $37 \%, 12.5 \%$ and $19 \%$, respectively, at 18 years. Although a tendency to lower terminal flow was seen in the girls, the differences were not statistically significant. Significant correlations between pulmonary function and the airway colonizator or genotype were not detected statistic. Conclusion: The evolution pattern of pulmonary function alterations found in these patients is consistent with those reported in the literature, where obstructive ventilatory disorders with early terminal flow reduction and late FVC onset predominate.

Descritores - Espirometria. Fibrose cística. Ventilação pulmonar. Key words - Spirometry. Cystic fibrosis. Pulmonary ventilatory.

\section{INTRODUÇÃO}

Fibrose cística do pâncreas (FC), ou mucoviscidose, é uma doença genética de caráter autossômico recessivo com evolução fatal e que compromete o funcionamento de praticamente todos os órgãos e sistemas do organismo através da alteração da função das glândulas exócrinas. As principais repercussões clínicas decorrem do envolvimento das glândulas exócrinas pulmonares, que geram secreções espessas e pegajosas. Calcula-se que cerca de $90 \%$ dos pacientes morrem devido à progressão da doença pulmonar ${ }^{(1)}$.

Fibrose cística é causada pela mutação de um gene no braço longo do cromossoma 7, lócus 7q32, responsável pela produção da proteína cystic fibrosis transmembrane regulator (CFTR). Até o momento, mais de 700 mutações já foram descritas. O mecanismo fisiopatológico primário na FC é causado por uma anormalidade no transporte do íon cloro nas células epiteliais através da CFTR, que é, na realidade, o próprio canal de cloro. Nas glândulas exócrinas pulmonares o fluxo de cloro alterado gera um ambiente no interior dos canais exócrinos menos hidratado, pois secundariamente o fluxo de água através das células também se altera. As secreções produzidas são viscosas, aderentes e ionicamente diferentes, dificultando o transporte (clearance) mucociliar da via aérea e facilitando infecções persistentes, principalmente por germes como Pseudomonas aeruginosa e Staphylococcus aureus. Atualmente, acredita-se que a alteração iônica das secreções pulmonares iniba a função antimicrobiana peptídica e reduza a resistência a patógenos bacterianos,
Siglas e abreviaturas utilizadas neste trabalho

CFTR - Cystic fibrosis transmembrane regulator CPT - Capacidade pulmonar total

CVF - Capacidade vital forçada

FC - Fibrose cística

$\mathrm{FEF}_{50}$ - Fluxo expiratório forçado a $50 \%$ da capacidade vital

$\mathrm{FEF}_{75}$ - Fluxo expiratório forçado a $75 \%$ da capacidade vital

FMEF - Fluxo médio expiratório forçado

HCPA - Hospital de Clínicas de Porto Alegre

PFE - Pico de fluxo expiratório

$\mathrm{VEF}_{1}$ - Volume expiratório forçado no primeiro segundo

$\mathrm{VEF}_{1} / \mathrm{CVF} \%$ - Razão entre volume expiratório forçado no primeiro segundo e capacidade vital forçada

VR - Volume residual

versão molecular mais aceita para justificar a colonização/infecção bacteriana crônica nos pulmões dos císti$\cos ^{(2)}$.

A presença de secreções espessas e infectadas leva à obstrução das pequenas vias aéreas e desencadeamento de um processo inflamatório crônico. A inflamação, presente inclusive em pulmões anatomicamente normais de recém-nascidos, leva à formação de bronquiectasias e lesão pulmonar com progressão, em última instância, para insuficiência respiratória e morte. Clinicamente, o comprometimento pulmonar pode manifestar-se por bronquiolite, bronquite, atelectasias, bronquiectasias, pneumotórax, hemoptise, pneumonias recorrentes, cor pulmonale $e$ insuficiência respiratória ${ }^{(3)}$.

O distúrbio ventilatório na FC é essencialmente obstrutivo. Somente na fase final surge um componente restritivo devido à fibrose pulmonar associada. As alterações da função pulmonar mais precoces refletem o acometimento inicial nas vias aéreas periféricas, demonstrado por diminuição dos fluxos expiratórios terminais e alçapona- 
mento aéreo, conforme evidenciado por diminuição do $\mathrm{FEF}_{50}, \mathrm{FEF}_{75}$ e FMEF, diminuição da relação $\mathrm{VEF}_{1} / \mathrm{CVF} \%$ e aumento da relação VR/CPT. Na fase tardia, a fibrose pulmonar leva à redução dos volumes pulmonares, mas com persistência da importante obstrução das vias aéreas, gerando uma configuração da curva fluxo-volume muito característica da FC - pico inicial correspondendo ao PFE seguido de uma concavidade acentuada e CVF reduzida ${ }^{(4,5)}$ (Figura 1).

Como grupo, e ao longo de alguns anos, o declínio da função pulmonar sofre uma queda exponencial, sendo o $\mathrm{FEF}_{50}$ O parâmetro a mostrar as alterações mais precoces e significativas ${ }^{(6)}$. Nos quadros agudos de exacerbação pulmonar, $\mathrm{O} \mathrm{VEF}_{1}$ e a CVF podem sofrer marcadas diminuições, mas que são recuperadas com o tratamento ${ }^{(5)}$. Portanto, medidas individuais da função pulmonar podem ser úteis para avaliar a extensão da anormalidade, a progressão da doença e a resposta individual ao tratamento. As medidas seriadas podem revelar tendências quanto à progressão da doença ${ }^{(7)}$.

Com o intuito de avaliar o padrão da evolução dos fluxos e volumes pulmonares do grupo de pacientes com fibrose cística em acompanhamento na Unidade de Pneumologia Infantil e Serviço de Pneumologia do Hospital de Clínicas de Porto Alegre, realizamos uma análise retrospectiva dos testes de função pulmonar. Além disso, pretendemos analisar a relação entre a gravidade do comprometimento ventilatório com a idade, sexo e colonização pulmonar.

\section{MATERIAL E MÉTODOS}

Neste estudo foi realizada uma revisão dos prontuários dos pacientes portadores de fibrose cística, confirmada por duas dosagens de cloro no suor elevadas e/ou pesquisa genética com homozigose para mutação delta F508, em acompanhamento na Unidade de Pneumologia Infantil e Serviço de Pneumologia do HCPA desde 1987. Do prontuário foram extraídas informações sobre idade atual, idade ao diagnóstico, pesquisa genética, colonização brônquica $e$ os valores absolutos e percentuais para fluxos $e$ volumes da melhor espirometria de cada ano. Foram incluídos pacientes com idade superior a quatro anos que tivessem realizado exames de espirometria aceitáveis, segundo critérios do I Consenso Brasileiro de Espirometria ${ }^{(17)}$. Todos os exames haviam sido realizados na Uni-

Figura 1 - Exemplos de curvas fluxo-volume em pacientes com FC conforme idade e evolução da doença: A) menina, sete anos: curva fluxo-volume normal; B) menina, 16 anos: curva fluxo-volume com acentuação da concavidade da alça expiratória, CVF normal; C) muIher, 23 anos: curva fluxo-volume com pico inicial correspondendo ao PEF seguido de acentuada concavidade e CVF reduzida.

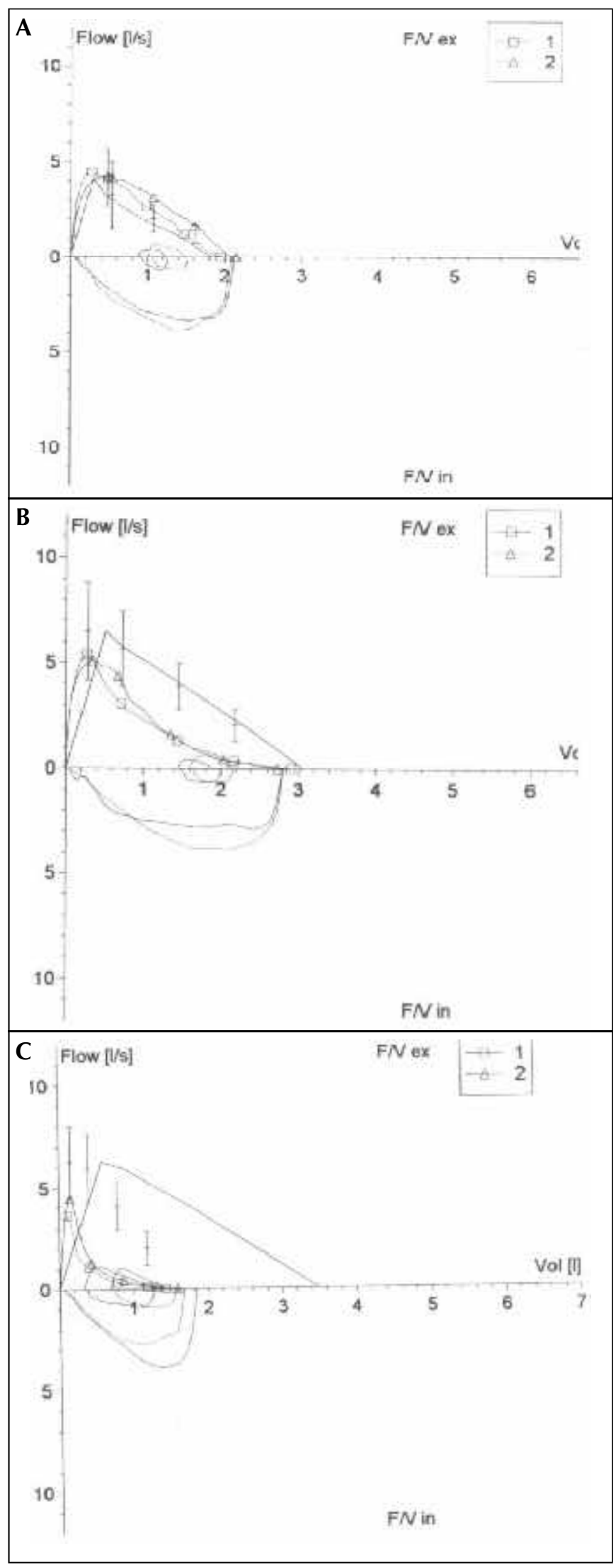

J Pneumol 27(3) - mai-jun de 2001 
dade de Fisiologia Pulmonar do HCPA e incluem espirometria simples e após broncodilatador, com medidas da $\mathrm{CVF}, \mathrm{VEF}_{1}, \mathrm{VEF}_{1} / \mathrm{CVF} \%, \mathrm{FEF}_{50}, \mathrm{FEF}_{75}$, FMEF e PFE.

Foram incluídos exames de pacientes com grau de acometimento pulmonar variável. Durante períodos de colonização com Burkolderia cepacia, por motivos de isolamento, os pacientes não puderam realizar testes de função pulmonar, retornando a realizá-los após um ano com cultura de escarro sem $B$. cepacia.

Estudos genéticos para pesquisa da mutação genética somente foram realizados em alguns pacientes para fim diagnóstico, ou seja, nos casos com valores de eletrólitos no suor limítrofes. Somente a mutação delta F508, presente em $90 \%$ dos casos de FC, era pesquisada.

Os aspectos éticos deste estudo referiram-se fundamentalmente a questões de sigilo e anonimato, tendo sido o mesmo submetido previamente à avaliação por um comitê de ética em pesquisa. Na análise estatística foram utilizados análise de variância e teste de Tukey para comparações múltiplas, considerando-se significativo o valor de $\mathrm{q} \alpha=0,05$.

\section{REsUlTADOS}

Foram avaliados 243 exames de 52 pacientes císticos realizados no período de 1987 a 1999. A idade dos pacientes variou de quatro a 26 anos (média de idade $=$ $13,04 \pm 4,82$ ). Destes, 17 eram meninas (33\%) e 35 meninos (67\%); dois haviam falecido. Um menino faleceu aos dez anos após ter sido acompanhado desde os cinco anos de idade, ocasião em que foi firmado o diagnóstico; uma menina faleceu aos 11 anos de idade, após tratamento desde os dois anos e seis meses. Os pacientes contribuíram com no mínimo uma espirometria, que em geral correspondia à primeira avaliação da função pulmonar em idade compativel com a sua correta execução ou à primeira após o diagnóstico estabelecido e, no máximo, com dez espirometrias. Na média, cada paciente contribuiu com 4,60 $\pm 2,62$ espirometrias para o total de 243 exames.

A média de idade ao diagnóstico foi de 2,95 $\pm 3,11$ anos. A idade média da realização da primeira espirometria foi de 7,48 anos ( $\pm 2,84$ anos) e o tempo médio transcorrido entre o diagnóstico e a primeira espirometria foi de 4,71 anos ( \pm 3,0 anos). Dos 52 pacientes, 49 (94\%) tiveram pelo menos uma cultural de escarro com Pseudomonas aeruginosa, 45 (86\%) com Staphylococcus aureus e 13 (25\%) com Burkolderia cepacia. Oito pacientes (15\%) eram homozigóticos para mutação delta F508 (Tabela 1).

O tempo de acompanhamento analisado variou de no mínimo dois anos a no máximo 11 anos (média de 4,58 $\pm 3,23$ anos). Na avaliação inicial na faixa etária dos qua- tro aos seis anos ( $\mathrm{n}=40)$, o valor médio da CVF foi de $114,24 \%$, mantendo-se acima de $80 \%$ até os 18 anos (n = 10), quando apresentou queda brusca do valor médio que atingiu $67,2 \%(p=0,0002)$. A média do $\mathrm{VEF}_{1}$ dos quatro aos seis anos foi de $112,25 \%$, apresentando queda abaixo de $80 \%$ aos dez anos ( $\mathrm{n}=23)$, atingindo $50 \%$ aos 18 anos $(\mathrm{p}<0,00001)$. A relação $\mathrm{VEF}_{1} / \mathrm{CVF} \%$ desde a avaliação pulmonar inicial apresentou-se reduzida, considerando-se um valor esperado de $86 \%$. O valor médio dos quatro aos seis anos foi de 85, com queda lenta progressiva, atingindo $63 \%$ aos 18 anos $(p=0,005)$. Dos quatro aos seis anos o valor médio do $\operatorname{FEF}_{50}(\mathrm{n}=26)$ foi de $94 \%$, atingindo $62 \%$ aos 11 anos $(n=17)(p=0,00005)$ e mostrando queda acentuada aos 14 anos $(\mathrm{n}=14)$, quando chegou aos $42 \%$ ( $p=0,063$ ), atingindo $37 \%$ aos 18 anos $(\mathrm{p}=0,010)$. O $\mathrm{FEF}_{75}$ médio inicial foi de $80 \%$, aos sete anos ( $\mathrm{n}=29)$ foi de $58 \%$ ( $\mathrm{p}=0,786)$ e já aos dez anos foi de $35 \%$ ( $p=0,002)$, chegando a $12,5 \%$ aos 18 anos $(p=0,021)$. O FMEF inicial foi de $90 \%$, caindo para cerca de $75 \%$ dos sete aos oito anos e atingindo média abaixo de $60 \%$ aos dez anos ( $p<0,00001)$. Após os 18 anos o FMEF médio foi muito baixo, chegando a $19 \%$ do previsto.

Os valores médios do $\mathrm{FEF}_{50}, \mathrm{FEF}_{75}$ e FMEF das meninas foram mais baixos e com queda mais acentuada do que os dos meninos até a adolescência, após a qual estes valores foram levemente superiores ou se igualaram. Estas

TABELA 1

Características da população estudada

\begin{tabular}{lrr}
\hline \multicolumn{1}{c}{ Variável } & $\mathbf{N}$ & $\mathbf{\%}$ \\
\hline Exames por idade $(\mathrm{n}=243)$ & & \\
4 a 6 anos & 40 & 16,46 \\
7 a 8 anos & 58 & 23,86 \\
9 a 10 anos & 43 & 17,69 \\
11 a 12 anos & 30 & 12,34 \\
13 a 14 anos & 26 & 10,69 \\
15 a 16 anos & 22 & 9,05 \\
17 a 18 anos & 14 & 5,76 \\
Mais de 18 anos & 10 & 4,11 \\
Sexo (n = 52) & & \\
Meninos & 35 & 67,31 \\
Meninas & 17 & 32,69 \\
Mutação genética & & \\
delta F508/delta F508 & 9 & 17,30 \\
delta F508/outra & 14 & 26,92 \\
Outra/outra & 8 & 15,38 \\
Não realizado & 21 & 40,38 \\
Colonização brônquica & & \\
Pseudomonas aeruginosa & & \\
Staphylococcus aureus & 49 & 94,23 \\
Burkolderia cepacia & 42 & 80,76 \\
\hline & 11 & 21,15 \\
\hline
\end{tabular}



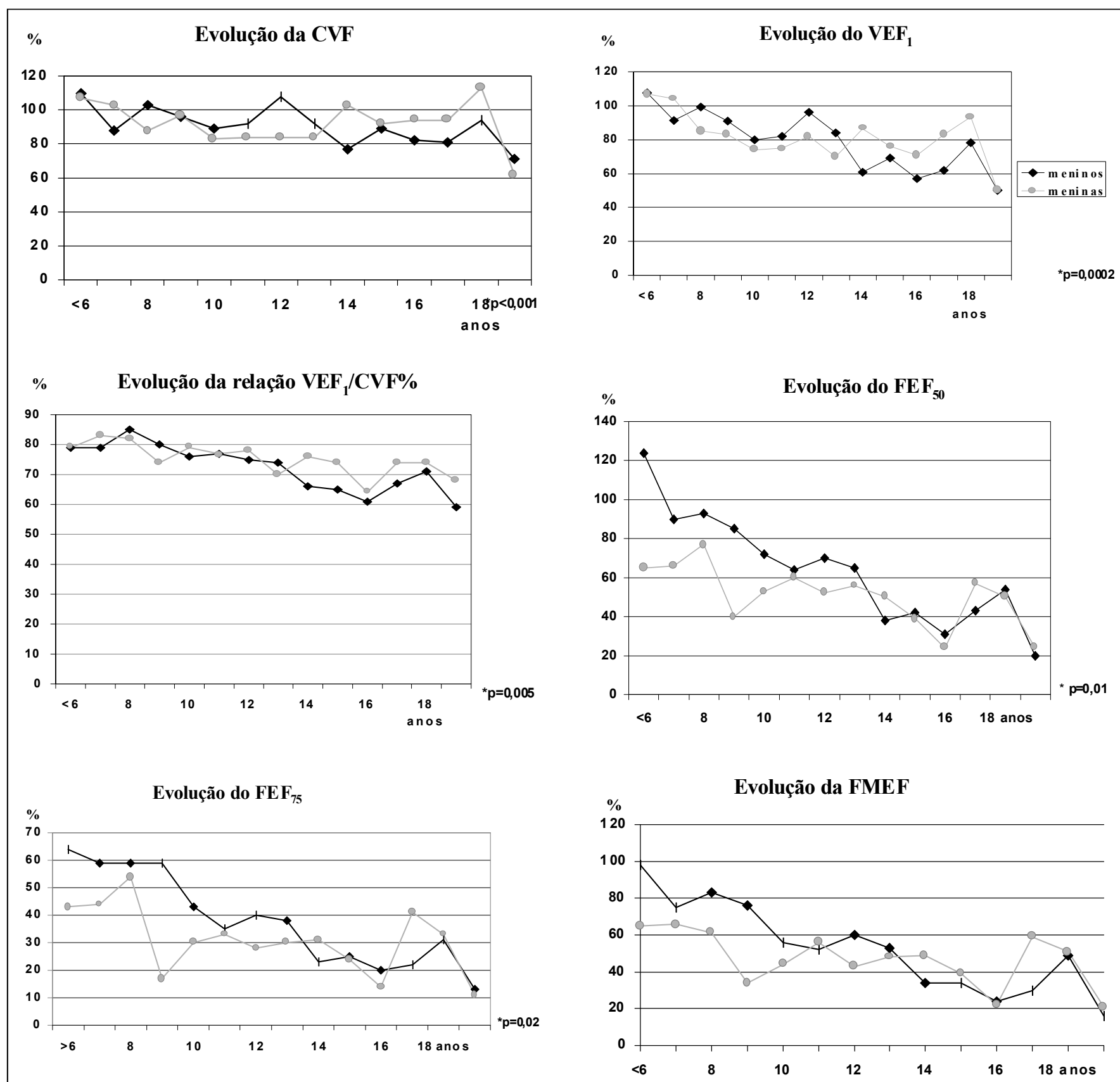

Evolução da PFE

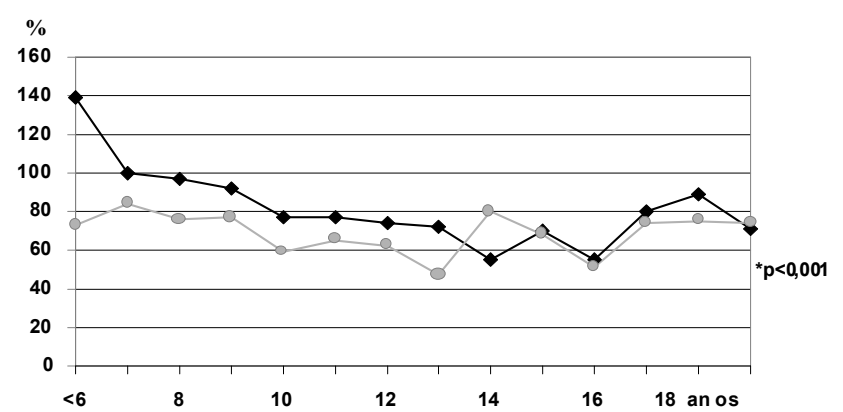

Figura 2 - Evolução do $V E F_{1}, C V F, V E F / C V F \%$, $F E F_{50}, F E F_{75}$, FMEF e PFE nos pacientes com fibrose cística 
diferenças não foram estatisticamente significativas, provavelmente porque no grupo havia mais meninos do que meninas. Entretanto, essas diferenças foram confirmadas em outros trabalhos.

Também não foram encontradas correlações estatisticamente significativas entre a função pulmonar e o germe colonizador da via aérea ou o genótipo, possivelmente devido ao pequeno número de pacientes.

A resposta ao broncodilatador não pôde ser aferida no nosso estudo porque a medicação $e$ a dose utilizadas variaram durante o período estudado.

\section{DISCUSSÃO E CONCLUSÃO}

As alterações funcionais mais importantes descritas na fibrose cística são: obstrução das vias aéreas, alçaponamento aéreo e ventilação inadequada. Medidas de ventilação-perfusão geralmente demonstram áreas focais de desequilíbrio. As alterações mais precoces na função pulmonar são alçaponamento de ar, que é evidenciado por aumento na relação volume residual/capacidade pulmonar total (VR/CPT) e diminuição dos fluxos expiratórios dos pequenos volumes pulmonares, ou seja, diminuição do $\mathrm{FEF}_{50}, \mathrm{FEF}_{75}$ e $\mathrm{FMEF}^{(5-9)}$. A quantidade de ar alçaponado em áreas pobremente ventiladas dos pulmões correlaciona-se negativamente com a condição clínica, ou seja, pacientes em piores condições tendem a ter maior alçaponamento aéreo ${ }^{(8)}$. Os demais índices da função pulmonar, como $\mathrm{CVF}, \mathrm{VEF}_{1}, \mathrm{VEF}_{1} / \mathrm{CVF}$, diminuem com a progressão da doença. $\mathrm{O} \mathrm{VEF}_{1}$, contudo, é um teste relativamente insensivel para avaliar a doença pulmonar na fase inicial $e$ pode inclusive ser normal mesmo quando há doença pulmonar clinicamente significativa ${ }^{(10)}$. Dessa forma, os testes de função pulmonar tornaram-se um instrumento de valor não só no monitoramento da perda da função pulmonar ao longo do tempo como também na monitorização da melhora da função pulmonar em resposta às várias estratégias terapêuticas empregadas ${ }^{(7,11,12)}$.

Num recente estudo epidemiológico de caráter multicêntrico, longitudinal e observacional (ESCF), realizado por Morgan et al., incluindo 18.411 pacientes císticos no Canadá e Estados Unidos, dados da função pulmonar, entre outros, foram coletados no período de dezembro de 1993 a dezembro de $1995^{(14)}$. Neste grupo de císticos, a CVF média entre seis e 12 anos foi acima de $90 \%$ do previsto; entre 13 e 17 anos manteve-se em 90\%, 80\% no grupo etário de 18 a 24 anos, atingindo $70 \%$ na faixa etária dos 25 aos 35 anos, mantendo-se em $70 \%$ aos 36 anos de vida ou mais. A média do $\mathrm{VEF}_{1}$ inicial na faixa de seis a 12 anos foi entre $85 \%$ e $90 \%$, com declínio um pouco mais acentuado, atingindo valores médios entre $75 \%$ e $80 \%$ na faixa dos 13 aos 17 anos, $60 \%$ dos 18 aos 24 anos, $50 \%$ dos 25 aos 35 anos, atingindo valor próximo aos $50 \%$ no sexo masculino e próximo aos $40 \%$ no sexo fe- minino na faixa dos 36 anos ou mais. Já a média do FMEF (ou $\mathrm{FEF}_{25-75}$ ) mostrou-se dos seis aos 12 anos próxima dos $70 \%$, com declínio mais rápido e precoce, atingindo valor médio de $50 \%$ entre 13 e 17 anos, $30 \%$ entre 18 e 24 anos, 20\% entre 25 e 35 anos e abaixo dos $20 \%$ aos 36 anos ou mais. Como esperado, $\mathrm{O} \mathrm{FEF}_{25-75}$ mostrou as reduções mais precoces, provavelmente refletindo o inicio da obstrução nas vias aéreas menores $e$ as meninas pareceram mostrar redução um tanto mais acentuada na função pulmonar em idades mais jovens que os meninos, relação esta que se igualou após a idade adulta, possivelmente devido ao efeito de sobrevida ${ }^{(13)}$.

Embora nosso estudo tenha a limitação de apresentar um número variável de pacientes ao longo das diferentes faixas etárias, envolver uma faixa etária menor e um número significativamente menor de registros, foi possivel identificar um grupo de pacientes císticos cuja evolução da função pulmonar segue o esperado, ou seja, redução precoce dos índices de ventilação das vias aéreas periféricas, refletindo o predomínio do distúrbio ventilatório obstrutivo, com perda mais acentuada destes parâmetros à medida que a doença pulmonar evolui e comprometimento tardio dos demais fluxos e da CVF.

Dados semelhantes foram encontrados no trabalho realizado por Beardsmore ${ }^{(7)}$, no qual medidas da mecânica pulmonar realizadas na idade de um a 24 meses (média de seis meses) foram comparadas com medidas do mesmo paciente na faixa etária dos quatro anos e dez meses a sete anos e cinco meses (média de cinco anos e dez meses). Enquanto a função pulmonar foi normal na maioria dos lactentes, na idade escolar a maioria apresentou deterioração dessa função, embora alguns continuassem a ter medidas normais. Não houve diferença significativa quanto aos valores de resistência da via aérea, mas a comparação entre os valores médios do volume de gás torácico nas duas faixas de idade foi significativa, assim como a comparação entre o $\mathrm{V}_{\max } \mathrm{FRC}$ na primeira infância e os fluxos terminais $\left(\mathrm{FEF}_{50}\right.$ e $\left.\mathrm{FEF}_{25}\right)$ na idade escolar, mostrando tendência à hiperinsuflação e desenvolvimento de obstrução nas pequenas vias aéreas, ao longo de cinco anos de observação.

O acometimento principal dos fluxos terminais já havia sido relatado por Zapletal et al. ${ }^{(9)}$, em 1971, e Corey et al. ${ }^{(6)}$, em 1976. Zapletal et al., nos primórdios da curva fluxo-volume, a identificaram como um teste sensivel $e$ quantitativo para monitoramento da obstrução das vias aéreas menores, tanto na FC quanto na asma. Corey e seus colegas revisaram as espirometrias de 132 pacientes com FC seguidos por cinco a sete anos e descreveram o declínio exponencial da função pulmonar dos císticos, sendo o $\mathrm{FEF}_{25-75 \%}$ a mostrar as alterações mais precoces e dramáticas, calculando-se uma queda de cerca de $8 \%$ ao ano. Entretanto, um pequeno grupo de císticos manteve 
a função pulmonar completamente normal durante o período estudado, indicando que talvez nem todos os pacientes apresentem um declínio progressivo na função pulmonar, o que provavelmente indicasse variantes mais leves da doença ${ }^{(6)}$.

Entre as possiveis variantes poderia estar implicado o genótipo, vistas as inúmeras mutações já descritas para FC. Entretanto, com exceção para insuficiência pancreática e a mutação delta F508, não se tem encontrado nenhuma correlação entre fenótipo e genótipo ${ }^{(14)}$. Tem-se demonstrado que alguns fatores externos podem influenciar o curso clínico da FC, entre os quais a infecção bacteriana, principalmente por Pseudomonas aeruginosa ${ }^{(15)}$, e exposição à fumaça de cigarro(16). No nosso estudo não foi possível mostrar diferenças na função pulmonar conforme a colonização bacteriana, visto que a maioria dos pacientes $(94,23 \%)$ já mostrava colonização por $P$. aeruginosa. Da mesma maneira, a análise da influência do genótipo fica também prejudicada, visto que $40,38 \%$ dos pacientes não haviam realizado o estudo genético.

Uma análise mais ampla da função pulmonar na fibrose cística permite identificar três padrões evolutivos: um

\section{REFERÊNCIAS}

1. Ramsey BW. Management of pulmonary disease in patients with cystic fibrosis. N Engl J Med 1996;335:179-188.

2. Davidson DJ, Porteous DJ. The genetics of cystic fibrosis lung disease. Thorax 1998;53:387-389.

3. Phelan PD, Olinski A, Robertson CF. Respiratory illness in children $4^{\text {th }}$ ed. London: Blackwell Scientific Publications, 1994;207-251.

4. Murray JF, Nadel JA. Textbook of respiratory medicine. $2^{\text {nd }} e d$. Philadelphia: WB Saunders, 1994;1418-1450.

5. Loughlin GM, Eigen H. Pulmonary function. In: Respiratory disease in children: diagnosis and management. Baltimore: Williams \& Wilkins, 1994;263-290

6. Corey M, Levison H, Crozier D. Five- to seven-year course of pulmonary function in cystic fibrosis. Am Rev Respir Dis 1976;114:10851092.

7. Beardsmore CS. Lung function from infancy to school age in cystic fibrosis. Arch Dis Child 1995;73:519-523.

8. I Consenso Brasileiro de Espirometria. J Pneumol 1996;22:125-129.

9. Zapletal A, Motoyama EK, Gibson LE, Bouhuys A. Pulmonary mechanics in asthma and cystic fibrosis. Pediatrics 1971;48:64-72.

10. MacLusky I, Levison H. Cystic fibrosis. In: Chernick V, Boat TF, Kendig Jr EL, eds. Disorders of respiratory tract in children. 6th ed. Philadelphia: WB Saunders, 1998;838-882. primeiro padrão, em que os valores permanecem estáveis por muitos meses ou mesmo muitos anos; um segundo padrão, que inclui um período de estabilidade seguido por um ponto de quebra, a partir do qual ocorre declínio linear da função pulmonar; e um terceiro padrão, no qual o declínio linear e progressivo começa ou já está presente aos seis anos de idade ${ }^{(10)}$.

Concluímos, finalmente, que para os principais parâmetros estudados houve diferenças significativas entre os valores médios iniciais dos quatro aos seis anos e os valores aos 18 anos, demonstrando queda da função pulmonar ao longo do tempo. O padrão de perda da função pulmonar foi semelhante ao previamente descrito em outros trabalhos, em que predomina o distúrbio ventilatório obstrutivo com redução precoce dos fluxos correspondentes às pequenas vias aéreas e acometimento tardio da capacidade vital forçada.

\section{AgradeCIMENTOS}

Os autores agradecem aos colegas Dra. Daniela Silveira Ferreira, Dra. Marineide Britto e Dr. Jocelito V. Martinez pelo auxílio durante a fase de coleta dos dados.

11. Ramsey BW, Boat TF. Outcome measures for clinical trials in cystic fibrosis. Summary of a Cystic Fibrosis Foundation Consensus Conference. J Pediatrics 1994;124:177-192.

12. Rosemberg SM, Howatt Wf, Grum CM. Spirometry and chest roentgenographic appearance in adults with cystic fibrosis. Chest 1992; 101:961-964.

13. Redding GJ, Restuccia R, Cotton EK, Brooks JG. Serial changes in pulmonary functions in children hospitalized with cystic fibrosis. Am Rev Respir Dis 1982;126:31-36.

14. Morgan WJ, Butler SM, Johnson CA, et al. Epidemiologic study of cystic fibrosis: design and implementation of a prospective, multicenter, observational study of patients with cystic fibrosis in the US and Canada. Pediatr Pulmonol 1999;28:231-241.

15. Milla PJ. Cystic fibrosis: present and future. Digestion 1998;59:579588.

16. Kerem E, Corey M, Gold R, Levison H. Pulmonary function and clinical course in patients with cystic fibrosis after pulmonary colonization with Pseudomonas aeruginosa. J Pediatr 1990;116:714-719.

17. Gilliam H, Stenlund C, Ericssson-Hollsing A, Strandvik B. Passive smoking in cystic fibrosis. Respir Med 1990;84:289-291. 